\title{
A novel technique for the preparation of fine ceramic oxides
}

\author{
AMITA PATHAK and P PRAMANIK \\ Department of Chemistry, Indian Institute of Technology, Kharagpur 721 302, India \\ Abstract. In this paper, we put forward a novel and simple chemical route for the preparation \\ of fine ceramic oxides i.e. ferrites using triethylammonium carbonate as the precipitating agent. \\ The particles were studied and characterized by X-ray diffraction and scanning electron \\ microscopy. The emphasis is on the superiority of this technique over other chemical and \\ conventional routes.
}

Keywords. Ceramic oxide; spinel; triethylammonium carbonate.

\section{Introduction}

The compounds of $\mathrm{AB}_{2} \mathrm{O}_{4}$ type with spinel structure have been studied by several researchers (Keeffe 1961; Goodenough 1963; Blasse 1965; Ghare and Sinha 1968; Rao and Subba Rao 1970), as they exhibit interesting structural, electrical and magnetic properties which vary with the nature of ions, their charge and site distribution amongst tetrahedral and octahedral sites. These fine particles of ceramic oxides find application in the field of electrical and electronics industries. The conventional preparation of these ceramic powders depends entirely on solid-state reactions. Besides being painfully time-consuming, such processes are affected by serious limitations of large inter-grain diffusion of various atomic species leading to multiphasic final products with wide particle-size distribution. To overcome these, chemical routes such as gel-precipitation (Fletcher and Hardy 1968; Marcilly et al 1970; Delmon and Droguest 1974; Baythoun and Sale 1982), coprecipitation using oxalates and citrates etc (Carcilly et al 1970; Anderson and Sale 1979; Bednorz et al 1987; Capone et al 1987; Wang et al 1987) have gained acceptance in recent years for their ability to give finer sinteractive particles with higher density and improved homogeneity.

In this paper, we report a novel and simple technique for the preparation of such fine amorphous ceramic oxides. In this study it was aimed at producing ferrites of Ni(II), $\mathrm{Zn}$ (II), $\mathrm{Cu}$ (II) and $\mathrm{Co}(\mathrm{II})$ ions from their corresponding salt solutions using triethylammonium carbonate (organic carbonate) as the precipitating agent. The effect of polymer coating of the precursors over the particle-size of these oxides was also studied. The particles obtained were characterized by X-ray diffraction (XRD) patterns, energy-dispersive $X$-ray spectroscopy (EDAX) and scanning electron microscopy (SEM).

\section{Experimental procedure}

Mixtures of ferric salt solutions and the corresponding salt solutions of the desired ferrites were prepared. The compositions chosen were based on the stoichiometry of $\mathrm{NiFe}_{2} \mathrm{O}_{4}, \mathrm{ZnFe}_{2} \mathrm{O}_{4}, \mathrm{CuFe}_{2} \mathrm{O}_{4}$ and $\mathrm{CoFe}_{2} \mathrm{O}_{4}$. These mixtures were separately poured into an excess of freshly prepared solution of triethylammonium carbonate (Pramanik et al 1990), with continuous stirring. The precipitates obtained were filtered, washed and 
Uried. It was then ground and calcined at $900^{\circ} \mathrm{C}$ for $150 \mathrm{~min}$. The precursors were decomposed/oxidized to form the respective ferrites.

To study the effect of polymer adsorption over these oxides, the above procedure for the preparation of ferrites was repeated in the presence of $2.5 \%$ solution of polyvinyl alcohol (PVA) in the solution-mixture before precipitation.

The products obtained were characterized by XRD patterns (figures 1 and 2) recorded using a Philips $\mathrm{PW}-1710 \mathrm{X}$-ray diffractometer and $\mathrm{Cu}-\mathrm{K}_{\alpha}$ radiafion. The composition and formation of these ferrites were confirmed by EDAX using ÁN 10,000 EDAX link

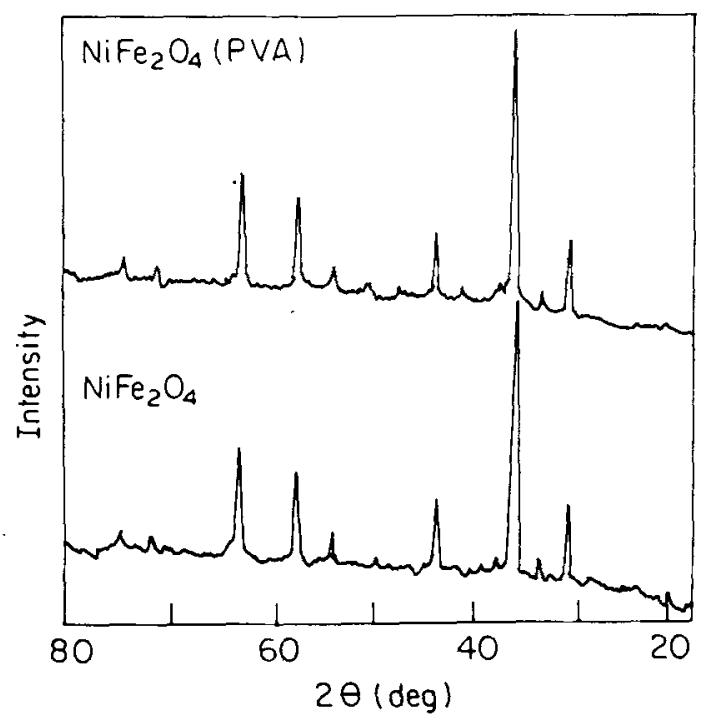

Figure 1. XRD pattern of $\mathrm{NiFe}_{2} \mathrm{O}_{4}$ in (a) absence of PVA and (b) presence of PVA.

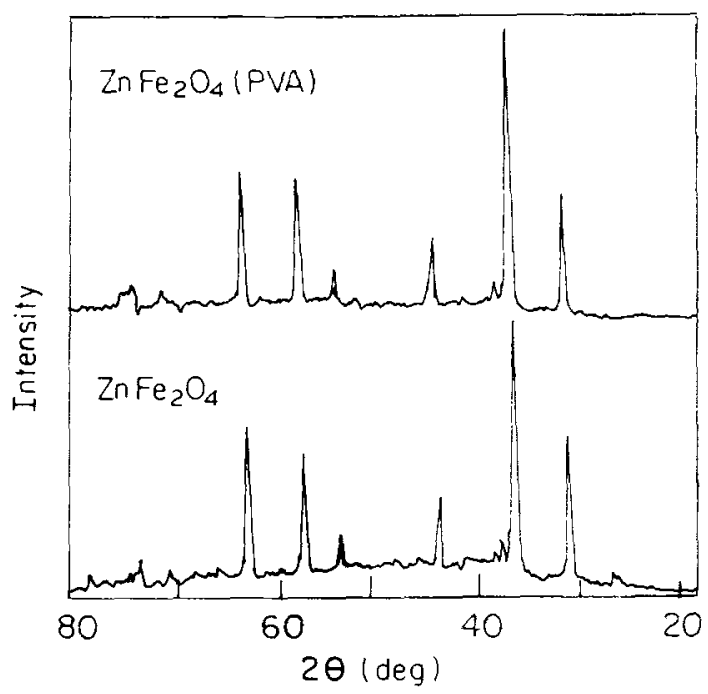

Figure 2. XRD pattern of $\mathrm{ZnFe}_{2} \mathrm{O}_{4}$ in (a) absence of PVA and (b) presence of PVA. 


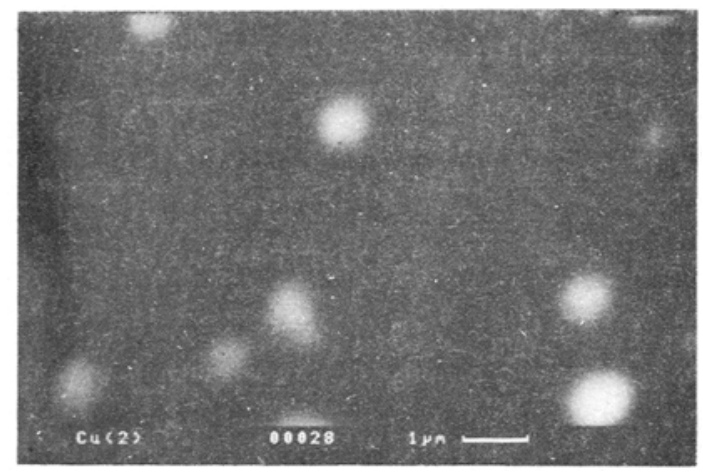

Figure 3. Scanning electron micrograph of $\mathrm{CuFe}_{2} \mathrm{O}_{4}$.

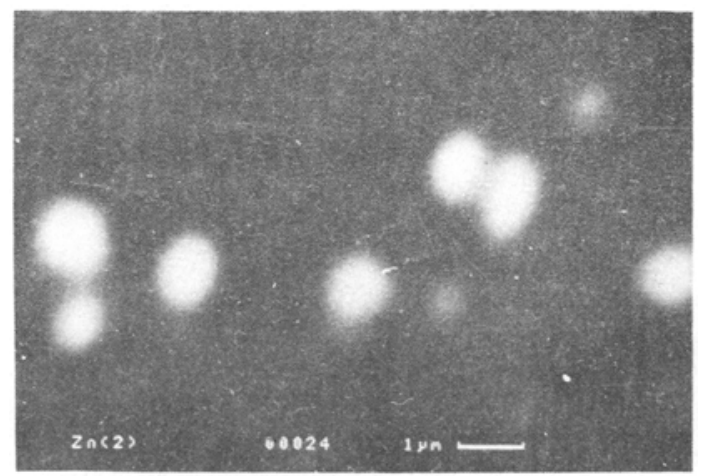

Figure 4. Scanning electron micrograph of $\mathrm{ZnFe}_{2} \mathrm{O}_{4}$,

system. SEM studies, using the model CAMSCAN series $2 \mathrm{DV}$, revealed the fine particle nature of the prepared ferrites (figures 3 and 4).

\section{Results and discussion}

The novelty of the organic carbonate precipitating technique over that of an inorganic carbonate process lies in its simplicity. These organic carbonates can be easily burnt off during calcination of the precursors to give the desired oxides, thus avoiding thorough washings. The inherent difficulty in using inorganic carbonates arises due to their persistence in the precursors and the laborious chemical treatment involved in their removal. Though the use of ammonium carbonate as a precipitating agent is an exception to this, our studies showed that its tendency to leach $\mathrm{Ni}$ (II) and $\mathrm{Cu}$ (II) ions from the precursors restricts its use.

The comparative study of XRD patterns of the calcined oxides obtained in the presence of PVA and in the absence of the same, shows no significant change in the peak-intensity and the peak-width reflecting the fact that no marked change in particlesize occurs in the presence of PVA (figures 1 and 2). SEM studies confirm this fact. These observations may be attributed to the possibility that the triethylammonium carbonate solution when added to salt solutions, for the precipitation, gives a gelnetwork structure in which the precursors' nuclei gets embedded and the particle-size 
growth is restricted. As the organic carbonate solution itself acts like a polymeric reagent, the presence of a polymer coating on the precursors does not significantly serve the purpose of further restricting the particle-size growth.

SEM studies revealed the particle-size range to be between 1 and $3 \mu \mathrm{m}$. The observed densities of the ferrites varied from $95-97 \%$ from the theoretical densities. In conclusion, it has been possible to successfully obtain high yield of single-phasic, fine, amorphous, homogeneous ferrite particles with minimum chemical treatment using triethylammonium carbonate precipitating technique. Moreover this method is costeffective since triethylamine can be easily recovered from the filtrate for reuse. Dimethylammonium carbonate, trimethylammonium carbonate and diethylammonium carbonate may also be used in place of triethylammonium carbonate for the precipitation of ferrites and other mixed-oxide systems (Pramanik et al 1990).

\section{References}

Anderson D J and Sale F R 1979 Powder Metall. 114

Baythoun M S G and Sale F R 1982 J. Mater. Sci. 172757

Bednorz J G, Takashige M and Muller K A 1987 Europhys. Lett. 3379

Blasse G 1965 Philips Res. Rept. 20528

Capone D W, Hinks D G, Jorgensen J D and Zhang K 1987 Appl. Phys. Lett. 50543

Carcilly C, Courty P and Delmon B $1970 \mathrm{~J}$. Am. Ceram. Soc. 5356

Delmon B and Droguest $\mathrm{J} 1974$ in Fine particle-Second International Conference, (eds) W E Kuhu and J Ehretsman (New Jersey: The Electrochemical Society) p. 242

Fletcher J M and Hardy C J 1968 Chem. Ind. 8748

Ghare D B and Sinha A P B 1968 J. Phys. Chem. Solids 29865

Goodenough J B 1963 in Magnetism and chemical bond (New York, London: Wiley) p. 120

Keeffe M O 1961 J. Phys. Chem. Solids 21172

Marcilly C, Courty P and Delmon B $1970 \mathrm{~J}$. Am. Ceram. Soc. 5356

Pramanik P, Biswas S, Chakravarty S, Roul B K and Chopra K L 1990 Mater. Res. Bull. 25877

Rao C N R and Subba Rao G V 1970 Phys. Status Solidi A1 597

Wang $\mathrm{H} H$ et al 1987 Inorg. Chem. 261474 